\title{
Impact of COVID 19, an Indian nephrologist's perspective
}

\author{
Abhilash Chandra ${ }^{1}$, Namrata Rao ${ }^{1}$, Divya Srivastava ${ }^{2}$ \\ ${ }^{1}$ Department of Nephrology, Dr. Ram Manohar Lohia Institute of Medical Sciences, Vibhuti Khand, Gomti Nagar, Lucknow, India.Affiliation- Dr.RMLIMS, Lucknow, India \\ 2 Department of Anaesthesiology, Sanjay Gandhi Post Graduate Institute of Medical Sciences, Lucknow, India.
}

India saw its first case of corona virus disease (COVID) 2019 on 30 th January 2020 . With the virus expanding across continents, it was a certainty that India would also be affected. To prevent exponential growth of disease, a complete lockdown sans essential services (food and medical items) was announced by the government on 25th March for 21 days and later extended till 3rd of May, with conditional relaxations after 20th April depending upon the regional spread of the disease. ${ }^{1}$ After a series of steps to downscale lockdown measures, unlock was finally started in June. ${ }^{2}$

Specialty services here are primarily urban-centric. Cessation of public transport in lockdown period meant complete reliance on private transport and ambulances for medical services. Unlocking eased the availability of public conveyance but at the same time increased the risk of infection from severe acute respiratory syndrome corona virus 2 (SARS CoV 2). While unlocking helped ease the economic burden on the families of kidney disease patients, it also brought a series of challenges. Foremost was the increased chance of infection as social distancing in congested dwelling places and public transport was often not followed. Awareness regarding proper usage of mask was also poor as was evident with patients repeatedly touching its outer surface and using it for longer periods than recommended. In order to counter the risk, dialysis patients and their attendants underwent training on the proper usage of masks, hand washing, and social distancing. Use of public transport was asked to be avoided or limited to hospital visits with due precautionary measures. In the absence of clear guidelines, screening of all dialysis patients with RT-PCR every 14 days was performed as a part of standard operating procedure. Usage of face masks, caps, gloves, goggles, and gowns by the dialysis staff was encouraged along with frequent cleaning of the dialysis room and equipment with bleach and $70 \%$ alcohol. Few centers reported an outbreak of COVID. These centers had to be temporarily closed and the scheduled patients were temporarily shifted to other centers after the COVID positive patients were shifted to dedicated COVID hospitals screening.

In this scenario, peritoneal dialysis offered a safer option with limited travel requirement. Obtaining the PD fluid, however, was an issue as the patient or care giver was required to visit stockists located at a distance. Follow-up was primarily by telemedicine services started by the hospital.

Cessation of OPD services particularly affected CKD stage 5 patients who required initiation of renal replacement therapy. Explaining the emotional and financial implications of this requires face-to-face counselling sessions, something which was often left wanting. It is difficult to ascertain this unmet need till OPD facilities are restored.

Transplant surgeries which were restricted to emergency situations in selected patients were restarted in line with recommendations. ${ }^{3}$ Informed consent about the spread of COVID and its associated implications was obtained from the recipients. Local availability of immunosuppressants and likelihood of safe follow-ups with a nephrologist was checked. Separate dedicated teams for managing transplant surgery and the postoperative period were formed.

Routine ward admissions presented a peculiar picture. They had to be reduced because of diversion of the staff to the designated COVID hospitals. Around 20-30 renal biopsies used to be performed at our center every month. They became reduced to $3-4 / \mathrm{mth}$. during the lockdown period but have now regained $50 \%$ of their original count. The grim situation is that of subjects with rapidly progressive renal failure. A number of them could have lost their renal functions in the absence of timely intervention. Some may have untimely stopped their immunosuppressants.

With the compromised workforce and the diverted attention to COVID preparation and management, academics have taken a back seat.

Although the picture is gloomy at the moment, there is hope that with flattening of the viral curve in the coming days, some normalcy will be regained. We can only hope the wait has been worth the pain.

Disclosure of potential conflicts of interest: none declared

\section{References \\ 1. "PM Modi announces extension of lockdown till 3 May". Livemint. 14 April 2020. \\ 2. Sharma, Neeta (30 May 2020). Ghosh, Deepshikha (ed.). "'Unlock1": Malls, Restaurants, Places of Worship to Reopen June 8". NDTV. Retrieved 30 May 2020 \\ 3. ISOT Position Statement For Transplantation In Era Of COVID-19. Available at https://isot.co.in/ file/ISOT_V2_28_April_2020.pdf; Accessed July 26, 2020.}

\section{Correspondence to:}

\section{Abhilash Chandra, DM}

Department of Nephrology, Dr. Ram Manohar Lohia Institute of Medical Sciences, Vibhuti Khand, Gomti Nagar, Lucknow-226010, India

E-mail: acn393@gmail.com 